\title{
Liraglutide Improves Cardiovascular Risk as an Add-on to Metformin and Not to Insulin Secretagogues in Type 2 Diabetic Patients: A Real-life 48-Month Retrospective Study
}

\author{
Alessandro Ciresi · Enrica Vigneri - Stefano Radellini · Felicia Pantò • \\ Carla Giordano (iD
}

Received: September 27, 2017 / Published online: November 14, 2017

(c) The Author(s) 2017. This article is an open access publication

\begin{abstract}
Introduction: Although liraglutide is widely recognized to have glycemic and extra-glycemic effects, few studies have compared these effects in relation to hypoglycemic treatment starting from the diagnosis of diabetes. We evaluated the effectiveness of liraglutide in reducing the Framingham risk score (FRS) and visceral adiposity index (VAI) in relation to first-line hypoglycemic treatment from diagnosis of type 2 diabetes, continued without any changes.

Methods: We selected 105 diabetic outpatients who were treated with liraglutide for at least 48 months as an add-on therapy to metformin alone (group A, $n=52$ ) or insulin secretagogues (group B, $n=53$ ) from diagnosis time.
\end{abstract}

Results: Although both groups showed a reduction in BMI, waist circumference, blood pressure, HbA1c and triglycerides, only group A showed a significant reduction in FRS $(p<0.001)$ and VAI $(p=0.012)$ after 48 months. No significant intergroup difference was found

Enhanced content To view enhanced content for this article go to http://www.medengine.com/Redeem/ 74DCF0604094F3CC.

A. Ciresi · E. Vigneri · S. Radellini - F. Pantò

C. Giordano ( $\square)$

Section of Endocrinology, Biomedical Department of Internal and Specialist Medicine, University of Palermo, Palermo, Italy

e-mail: carla.giordano@unipa.it for any parameters at either baseline or 48 months, with the exception of FRS at 48 months, lower in group A $(p=0.002)$, regardless of duration of disease, improvement in glycemic control and VAI.

Conclusion: Our data show that during a 48-month follow-up liraglutide was more efficacious in reducing cardiovascular risk than when it was used as add-on therapy to the first-line therapy from diagnosis with metformin and not with insulin secretagogues.

Keywords: Cardiovascular risk; Diabetes; Liraglutide; Visceral adiposity

\section{INTRODUCTION}

Liraglutide is effective in the treatment of type 2 diabetes, showing both glycemic and extra-glycemic effects, thereby achieving many of the goals of ideal antihyperglycemic therapy [1]. The main effect is certainly a significant weight reduction, which seems to be independent of glycemic improvement [2].

In addition, a reduction of cardiovascular $(\mathrm{CV})$ risk was confirmed by large randomized trials [3], and the beneficial effect of liraglutide on visceral fat adiposity was clearly demonstrated [4]. The visceral adiposity index (VAI), a valuable indicator of visceral adipose function and insulin sensitivity, has been demonstrated to be an independent predictor of $\mathrm{CV}$ diseases 
and an easy tool for mirroring a condition of cardiometabolic (CM) risk [5].

This study aimed to evaluate the effectiveness of liraglutide in a real-world setting in reducing a composite index of $\mathrm{CV}$ risk [the Framingham risk score (FRS)] [4] and VAI in relation to first-line hypoglycemic treatment from the time of diagnosis of diabetes, which never changed during the entire period before liraglutide addition.

\section{METHODS}

In this retrospective study, we extracted data of 395 diabetic outpatients who were given liraglutide with at least 48 months of follow-up. Specifically, we carefully selected data of 135 patients who were treated with just one oral hypoglycemic agent continuously from diagnosis, excluding those whose therapy was modified even for a brief period. Before the 48-month observational period, no patient enrolled needed to have the hypoglycemic therapy modified on the basis of glycemic control (HbA1c levels), and there was good adherence until the last observation. During the 48 months of follow-up, any change in concomitant medications was considered an exclusion criterion. Specifically, 30 patients with a comparable number (14 in group A and 16 in group B) were excluded from the analysis (respectively 12 at 12 months, 6 at 24 months, 9 at 36 months and 3 at 48 months) because of discontinuation of liraglutide, a change in concomitant medications (9.6\%), low compliance with treatment $(8.8 \%)$ or lack of follow-up (3.7\%).

The final analysis comprised 105 patients, subdivided into group A [ 52 patients, mean age $55 \pm 8$ years, treated with liraglutide as an add-on therapy to metformin (MET)] and group B [53 patients, mean age $58 \pm 9$ years, treated with liraglutide on as an add-on to insulin secretagogues (SE)]. Due to the retrospective nature of the study and for the purpose of including all the patients who met the inclusion criteria expressed above, a priori power calculation was not performed.
In most cases, SE as first-line treatment was autonomously prescribed by primary care physicians of patients or as a result of MET severe gastric intolerance after the first 2-3 days and immediately suspended by patient's decision.

The FRS for estimating the 10-year risk for $\mathrm{CV}$ events was calculated on the basis of age, sex, total and HDL cholesterol, blood pressure and smoking status [6].

The VAI was calculated as described [5], using the following formulas:

Males: $\mathrm{VAI}=[$ waist circumference $/ 39.68$

$$
\begin{aligned}
& +(1.88 \times \text { body mass index })] \\
& \times(\text { triglycerides } / 1.03) \\
& \times(1.31 / \text { HDL cholesterol })
\end{aligned}
$$

Females: VAI $=[$ waist circumference $/ 36.58$ $+(1.89 \times$ body mass index $)]$ $\times($ triglycerides $/ 0.81)$ $\times(1.52 / \mathrm{HDL}$ cholesterol $)$.

All procedures were in accordance with the ethical standards of the responsible committee on human experimentation (institutional and national) and with the Helsinki Declaration of 1964, as revised in 2013. Approval was obtained from the Ethic Committee of the University of Palermo. At the time of hospitalization, an informed consent for the scientific use of the data was obtained from all patients for being included in the study.

\section{Statistical Analysis}

The SPSS version 19 (SPSS, Inc.) was used for data analysis. Data were presented as mean \pm SD or rates and proportions. The normality of distribution of the quantitative variables was assessed using the Kolmogorov-Smirnov test. The differences between the two groups were evaluated with $t$-Student for quantitative variables and $\chi^{2}$ for trend for categorical variables. A $p$ value $<0.05$ was considered statistically significant.

The statistical significance of the results was also confirmed through the data analysis of the "last observation carried forward" (LOCF) in the 
initial cohort of 135 patients, also including the latest clinical and biochemical data available at the last observation, corresponding to the time of exclusion from the study, of the 30 patients excluded from the final analysis.

\section{RESULTS}

Patients in group A and B were matched for duration of diabetes $(7.5 \pm 5.4 \quad$ vs. $8.6 \pm 5.2$ years; $p=0.305$ ), prevalence of hypertension $(42 \%$ vs. $43 \%$; $p=0.579)$, smoking status (38.4\% vs. $26.4 \% ; p=0.133$ ), previous CV events $(17.3 \%$ vs. $16.9 \% ; p=0.584)$, and use of antiplatelet therapy $(26 \%$ vs. $30 \% ; p=0.315)$ or statins $(27 \%$ vs. $20 \% ; p=0.103)$. Eighty-one percent of patients were taking antihypertensive drugs (angiotensin type 1 receptor blockers in 49 patients, angiotensin-converting enzyme inhibitors in 33 patients, calcium entry blockers in 16 patients, diuretics in 36 patients, beta-blockers in 30 patients), 53\% aspirin and 44\% statins, without any difference between the two groups. The mean dose of MET administered to patients in group A was $1.5-2.0 \mathrm{~g}$ daily. In group B, 25 (47\%) patients were taking repaglinide (3.0-4.0 g daily), 20 (38\%) gliclazide (240 mg daily) and 8 (15\%) glimepiride (4.0 mg daily). Liraglutide was administered at the dose of $1.2 \mathrm{mg}$ during the entire follow-up.

After addition of liraglutide, after 48 months all patients showed a significant reduction in body mass index $(p<0.001$ in both groups), waist circumference ( $p<0.001$ in both groups), systolic $(p<0.001$ in group $\mathrm{A} ; p=0.005$ in group $\mathrm{B})$ and diastolic blood pressure $(p<0.001$ in group $\mathrm{A} ; p=0.007$ in group $\mathrm{B}), \mathrm{HbA} 1 \mathrm{c}$ $(p<0.001$ in both groups) and triglycerides $(p<0.001$ in group $\mathrm{A} ; p=0.002$ in group $\mathrm{B})$, without any significant change in HDL and LDL cholesterol (Table 1).

In the whole population, the FRS showed a significant reduction from baseline to 48 months $(p=0.002)$, while, subdividing patients into the two groups, this result was confirmed in group A $(p<0.001)$ but not in group B $(p=0.077)$. Similarly, the VAI showed a significant reduction in the whole population $(p=0.002)$ and in group A $(p=0.012)$, but not in group B ( $p=0.058)$ (Fig. 1). At 48 months, group A showed a lower FRS $(p=0.002)$ and a higher change in FRS from baseline to 48 months $(p<0.001)$ than group $\mathrm{B}$, without any difference in other parameters (Table 1 ).

At multivariate analysis, concomitant hypoglycemic therapy proved to be the only variable independently associated with both FRS at 48 months $(\beta \quad 0.303 ; 95 \%$ CI $1.480-5.880$; $p=0.001)$ and the change in FRS from baseline to 48 months ( $\beta$ 0.424; 95\% CI $0.239-0.601$; $p<0.001$ ) (Table 2).

The statistical significance of these results was also confirmed when we performed the LOCF data analysis in the initial cohort of 135 patients (data not shown). No serious adverse or $\mathrm{CV}$ events were observed during the follow-up.

\section{DISCUSSION}

This study demonstrates a reduction in both FRS and VAI when liraglutide is added to metformin compared to SE, regardless of duration of disease and other clinical and biochemical parameters.

Liraglutide is known to be effective in reducing common $\mathrm{CV}$ risk factors [7-9]. In addition, the effectiveness of liraglutide in reducing leptin levels [10] and the VAI [11] has recently been evaluated. In the last few years, many studies have evaluated the capability of the VAI to express the CM risk and a possible adipose tissue dysfunction, in both the general population and diabetic patients $[12,13]$.

To date, few studies have been mainly directly aimed at comparing the extra-glycemic effects of liraglutide in relation to previous and concomitant hypoglycemic treatment, especially in the absence of modification of the association therapy starting from diagnosis of diabetes. In this study, although the individual $\mathrm{CV}$ risk factors seemed to be improved in the both groups, the FRS proved to be differently influenced by the two treatments, indicating a different role on $\mathrm{CV}$ played by liraglutide added to metformin or to SE. Due to the different behaviors of the parameters that affect FRS and VAI, only FRS was significantly different between the two groups at the end of follow-up, 


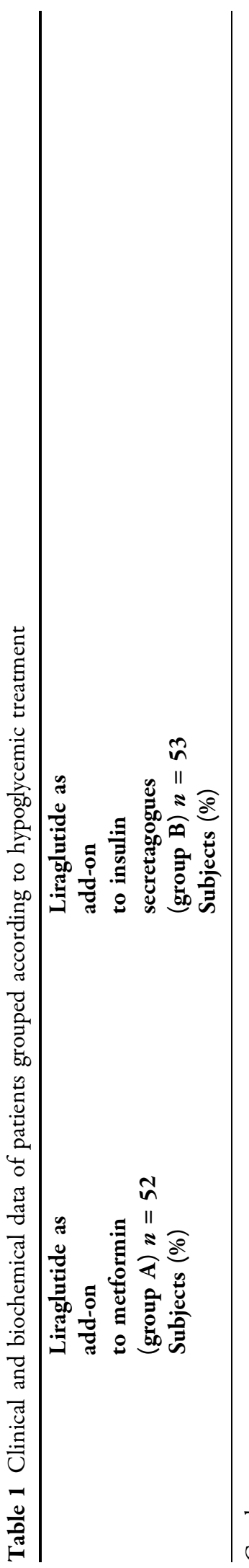

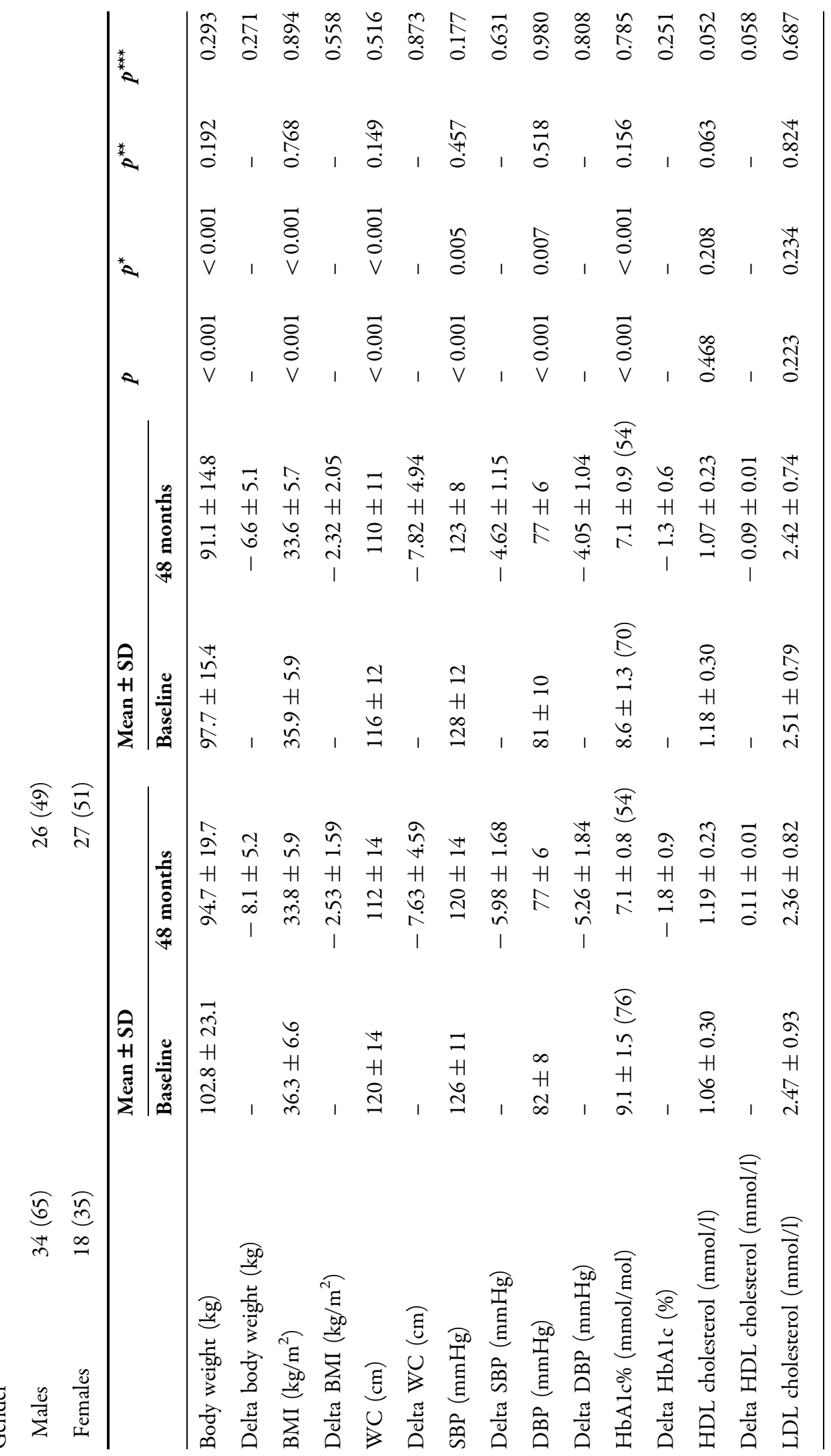




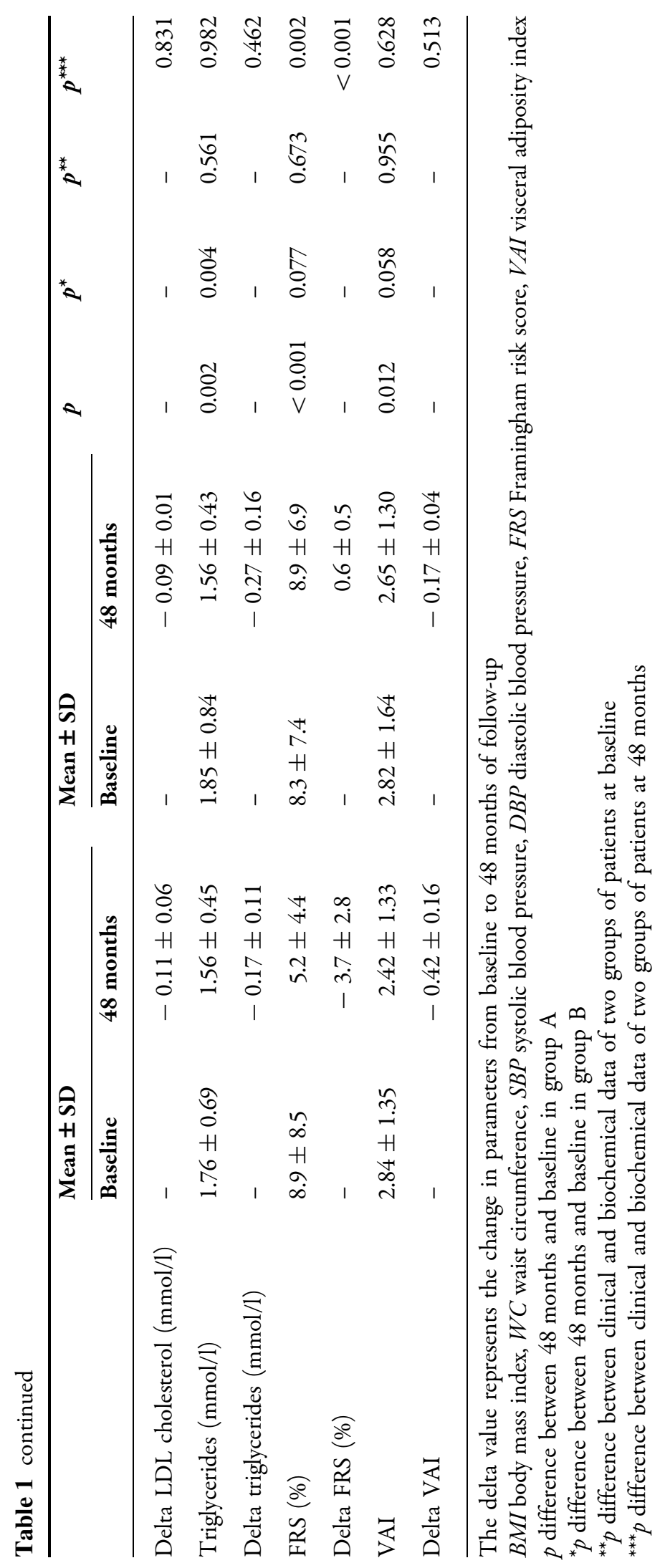


Reduction in VAI during the follow-up according to treatment
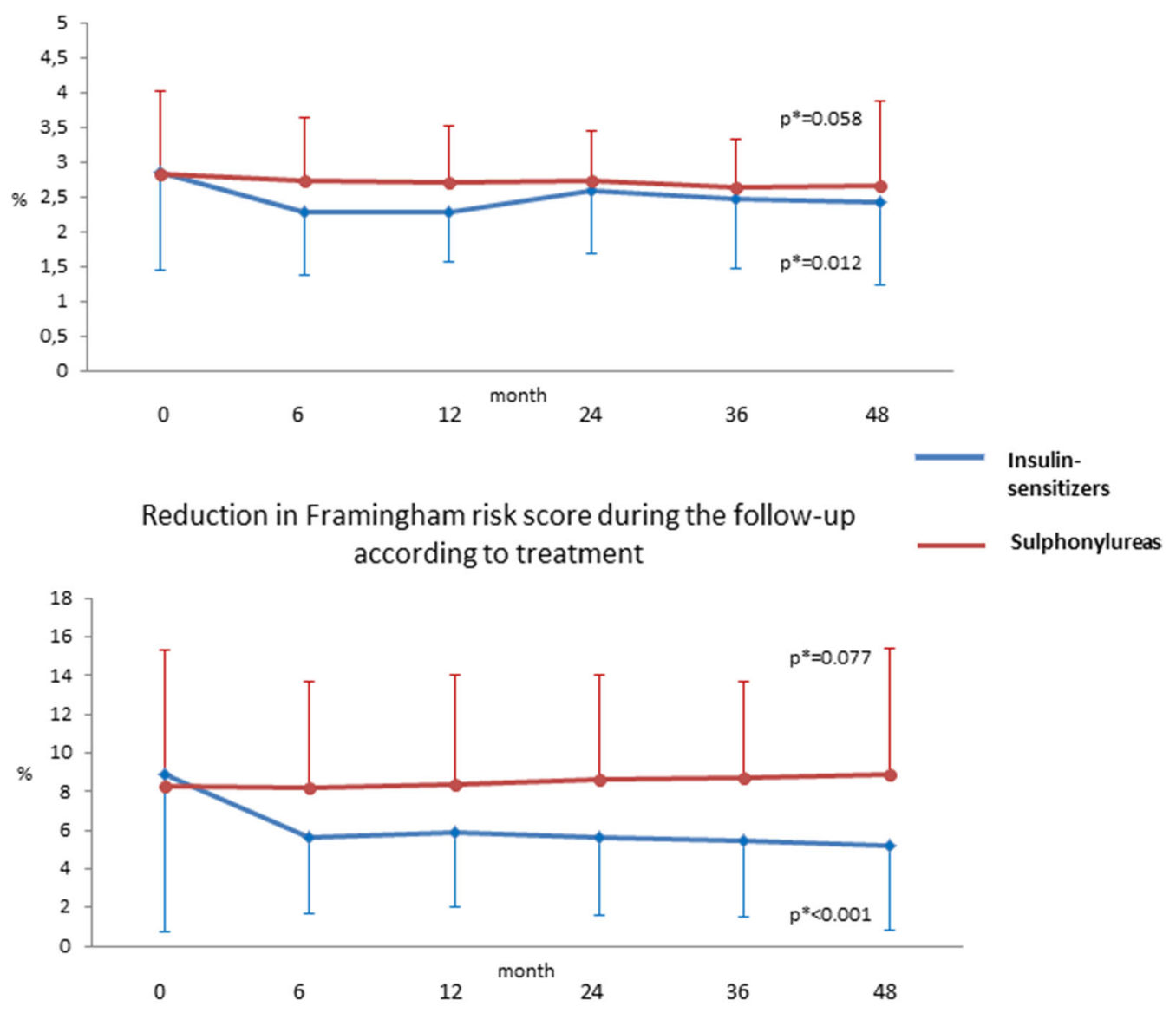

$\mathrm{p}^{*}=$ change from baseline to 48 months

Fig. 1 Change in visceral adiposity index (a) and Framingham risk score (b) during liraglutide treatment in diabetic patients grouped according to concomitant hypoglycemic therapy (blue line: metformin; orange line: insulin secretagogues)

showing a reduction of above $40 \%$ in the group A and a trend toward an increase, although not significant, in group B. In agreement with these data, it is widely demonstrated that the addition of liraglutide to metformin proves effective in improving several CV risk markers beyond glycemic control [14]. In our patients, the addition of liraglutide did not significantly change the natural clinical course of patients who took SE, while it significantly modified it when it was added to metformin.

Confirming this datum, in our study the concomitant hypoglycemic treatment was confirmed to be the only variable independently and strongly associated with the FRS at multivariate analysis.
We could hypothesize that the beneficial effect of liraglutide can be counterbalanced by the effects of SE, whereas they are emphasized by association with MET. These data are in agreement with a previous large retrospective observational study, which showed that the probability of reaching good glycemic control was significantly higher in patients previously treated with metformin alone and without any previous insulin, reinforcing the hypothesis that better results with liraglutide could be achieved in patients previously treated with metformin [15].

The potential mechanism of these different outcomes in the two groups is not fully clear, although it has been widely demonstrated that 


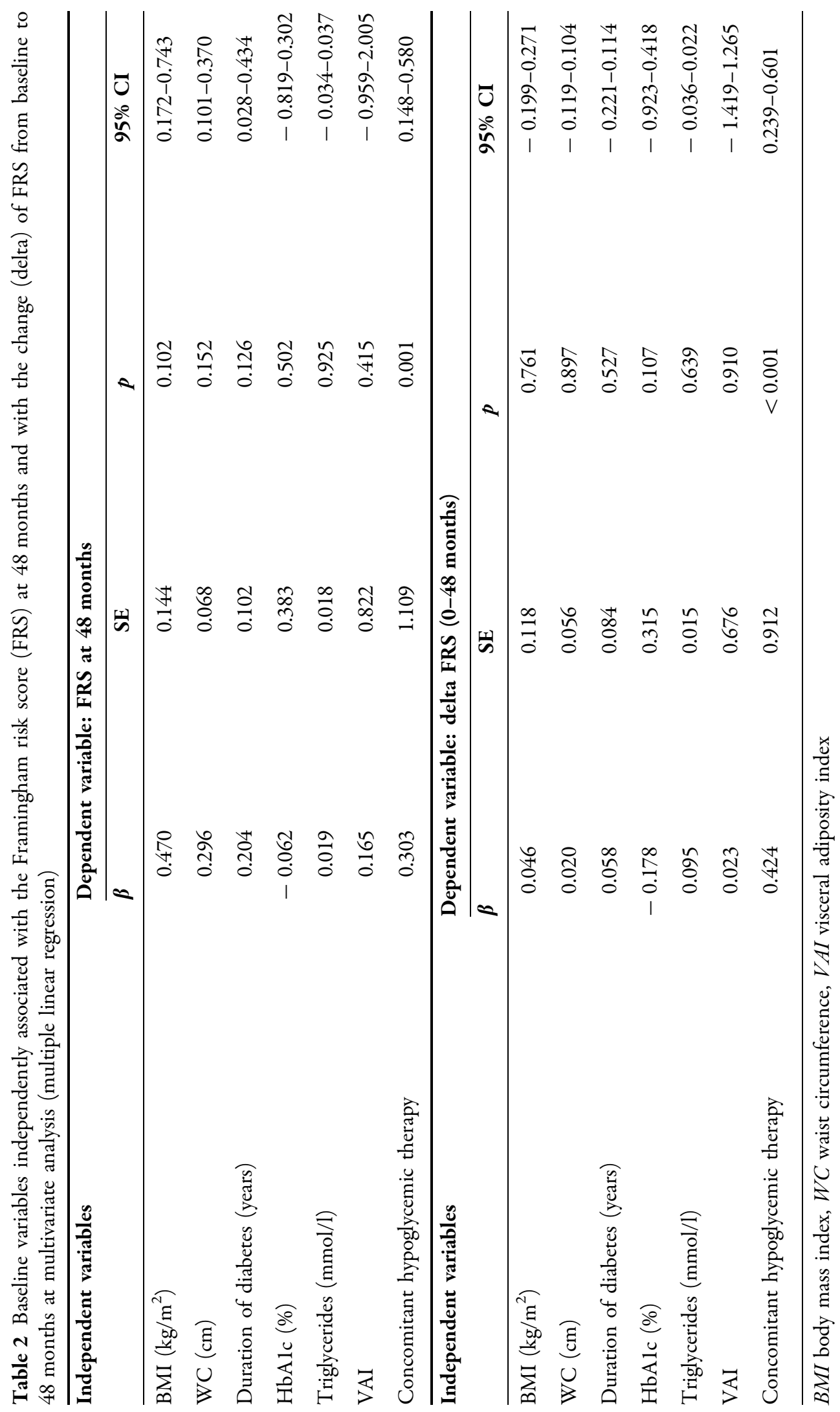


sulphonylurea and MET may differentially affect the CV risk in diabetic patients [16-18]. A hypothesis might be that sulphonylurea can progressively cause $\beta$-cell failure by reducing the efficacy of liraglutide [19].

It should be noted that the FRS is a tool for $\mathrm{CV}$ risk assessment in the general population with or without diabetes [6], so its application in a diabetic population could potentially represent a bias. Indeed, in the FRS glycemic control and duration of diabetes are not included, although these variables are important predictors of CV risk. In this study, neither glycemic control nor duration of disease may have played a role, as they were similar in the two groups, and the efficacy of liraglutide therapy in reducing HbA1c levels was similar in the two groups. Therefore, in this specific case the change in FRS may be considered an extraglycemic effect of liraglutide. In addition, by multivariate analysis we demonstrated that the specific parameters of diabetes do not have any significant influence on the outcome of the study (FRS at 48 months and delta FRS from baseline to 48 months). Of course, the diabetic population of our study possesses a higher well-recognized $\mathrm{CV}$ risk with respect to the general population. However, the aim of this study was not to cross-sectionally evaluate the $\mathrm{CV}$ risk in the diabetic population, obviously different from non-diabetic subjects, but to observe the effect of two different hypoglycemic treatments on longitudinal FRS modification.

A potential limitation of this study is the small size of the sample of diabetic patients in whom a priori power calculation was not performed because of the retrospective nature of the study. In this regard, we decided to rigorously select patients according to unchanged hypoglycemic treatment to have more reliable results and avoid interference with the outcomes of the study. Here, we show the results of a smaller but homogeneous diabetic population followed in a real-life setting, although certainly our data need to be confirmed in a larger series of patients.

In conclusion, our preliminary data extracted from real-life clinical practice suggest that liraglutide as an add-on therapy to metformin during a follow-up of 48 months is more efficient in reducing the FRS compared with addition to SE, regardless of the improvement in glycemic control and VAI.

\section{ACKNOWLEDGEMENTS}

No funding or sponsorship was received for this study or publication of this article. Article processing charges were funded by the authors. All named authors meet the International Committee of Medical Journal Editors (ICMJE) criteria for authorship for this manuscript, take responsibility for the integrity of the work as a whole and have given final approval to the version to be published. All authors had full access to all of the data in this study and take complete responsibility for the integrity of the data and accuracy of the data analysis.

Disclosures. Alessandro Ciresi, Enrica Vigneri, Stefano Radellini, Felicia Pantò and Carla Giordano have nothing to disclose.

Compliance with Ethics Guidelines. All procedures were in accordance with the ethical standards of the responsible committee on human experimentation (institutional and national) and with the Helsinki Declaration of 1964 , as revised in 2013. Approval was obtained from the Ethic Committee of the University of Palermo. At the time of hospitalization, an informed consent for the scientific use of the data was obtained from all patients for being included in the study.

Data Availability. The data sets generated during and/or analyzed during the current study are available from the corresponding author on reasonable request.

Open Access. This article is distributed under the terms of the Creative Commons Attribution-NonCommercial 4.0 International License (http://creativecommons.org/licenses/ by-nc/4.0/), which permits any noncommercial use, distribution, and reproduction in any medium, provided you give appropriate credit to the original author(s) and the source, 
provide a link to the Creative Commons license, and indicate if changes were made.

\section{REFERENCES}

1. Rigato M, Fadini GP. Comparative effectiveness of liraglutide in the treatment of type 2 diabetes. Diabetes Metab Syndr Obes. 2014;7:107-20.

2. Fadini GP, Simioni N, Frison V, Dal Pos M, et al. Independent glucose and weight-reducing effects of Liraglutide in a real-world population of type 2 diabetic outpatients. Acta Diabetol. 2013;50(6):943-9.

3. Marso SP, Daniels GH, Brown-Frandsen $\mathrm{K}$, et al. Liraglutide and cardiovascular outcomes in type 2 diabetes. N Engl J Med. 2016;375(4):311-22.

4. Inoue K, Maeda N, Kashine S, et al. Short-term effects of liraglutide on visceral fat adiposity, appetite, and food preference: a pilot study of obese Japanese patients with type 2 diabetes. Cardiovasc Diabetol. 2011;10:109.

5. Amato MC, Giordano C, Galia M, et al. Visceral Adiposity Index: a reliable indicator of visceral fat function associated with cardiometabolic risk. Diabetes Care. 2010;33(4):920-2.

6. D'Agostino RB Sr, Vasan RS, Pencina MJ, et al. General cardiovascular risk profile for use in primary care: the Framingham Heart Study. Circulation. 2008;117:743-53.

7. Nauck M, Frid A, Hermansen K, et al. Efficacy and safety comparison of liraglutide, glimepiride, and placebo, all in combination with metformin, in type 2 diabetes: the LEAD (liraglutide effect and action in diabetes)-2 study. Diabetes Care. 2009;32(1):84-90.

8. Seufert J, Gallwitz B. The extra-pancreatic effects of GLP-1 receptor agonists: a focus on the cardiovascular, gastrointestinal and central nervous systems. Diabetes Obes Metab. 2014;16(8):673-88.

9. Courrèges JP, Vilsbøll T, Zdravkovic M, et al. Beneficial effects of once-daily liraglutide, a human glucagon-like peptide- 1 analogue, on cardiovascular risk biomarkers in patients with type 2 diabetes. Diabet Med. 2008;25(9):1129-31.
10. Farr OM, Tsoukas MA, Triantafyllou G, et al. Short-term administration of the GLP-1 analog liraglutide decreases circulating leptin and increases GIP levels and these changes are associated with alterations in CNS responses to food cues: a randomized, placebo-controlled, crossover study. Metabolism. 2016;65(7):945-53.

11. Russo GT, Labate AM, Giandalia A, et al. Twelvemonth treatment with Liraglutide ameliorates visceral adiposity index and common cardiovascular risk factors in type 2 diabetes outpatients. J Endocrinol Investig. 2015;38(1):81-9.

12. Amato MC, Pizzolanti G, Torregrossa V, et al. Visceral adiposity index (VAI) is predictive of an altered adipokine profile in patients with type 2 diabetes. PLoS One. 2014;9(3):e91969.

13. Al-Daghri NM, Al-Attas OS, Alokail MS, et al. Visceral adiposity index is highly associated with adiponectin values and glycaemic disturbances. Eur J Clin Investig. 2013;43:183-9.

14. Forst $\mathrm{T}$, Michelson $\mathrm{G}$, Ratter $\mathrm{F}$, et al. Addition of liraglutide in patients with type 2 diabetes well controlled on metformin monotherapy improves several markers of vascular function. Diabet Med. 2012;29(9):1115-8.

15. Lapolla A, Frison V, Bettio M, et al. Correlation between baseline characteristics and clinical outcomes in a large population of diabetes patients treated with liraglutide in a real-world setting in Italy. Clin Ther. 2015;37(3):574-84.

16. Hong J, Zhang Y, Lai S, et al. Effects of metformin versus glipizide on cardiovascular outcomes in patients with type 2 diabetes and coronary artery disease. Diabetes Care. 2013;36(5):1304-11.

17. Phung OJ, Schwartzman E, Allen RW, Engel SS, Rajpathak SN. Sulphonylureas and risk of cardiovascular disease: systematic review and meta-analysis. Diabet Med. 2013;30(10):1160-71.

18. UK Prospective Diabetes Study (UKPDS) Group. Effect of intensive blood-glucose control with metformin on complications in overweight patients with type 2 diabetes (UKPDS 34). Lancet. 1998;352(9131):854-65.

19. Kozawa J, Inoue $\mathrm{K}$, Iwamoto $\mathrm{R}$, et al. Liraglutide is effective in type 2 diabetic patients with sustained endogenous insulin-secreting capacity. J Diabetes Investig. 2012;3(3):294-7. 